\title{
Solid-Phase Extraction of Caffeine and Catechin Compounds from Green Tea by Caffeine Molecular Imprinted Polymer
}

\author{
Yinzhe Jin and Kyung Ho Row \\ Center for Achanced Bioseparation Technolog: Deparment of Chemical Engineering, \\ Inha Linversity, Incheon $402-751$. Korea. ${ }^{*}$ E-mail: row kho ainha.ac.kr \\ Received October 17, 2006
}

\begin{abstract}
In this work. caffeine and some catechin compounds + C. EC. EGC. and EGCG were extracted from green tea by using molecular imprinted polymers (MIP) as sorbent materials in a solid-phase extraction (SPE) process known as MISPE (molecular imprinted solid-phase extraction). For ș'nthesis of MIP. caffeine was employed as the template. MAA as the monomer. EGDMA as the crosslinker. and AIBN as the initiator. A solution of caffeine $(0.2 \mathrm{mg} / \mathrm{mL}$ in methanol) was utilized in the solid extraction cartridges following loading. washing. and elution procedures with acetonitrile. methanol, and methanol-acetic acid $(90 / 10, \% \mathrm{v} / \mathrm{v})$ as the solvents. respectively: This solid-phase extraction protocol was applied for the extraction of caffeine and some catechin compounds from green tea. A comparison was made between the results obtained with the MIP cartridges and a traditional $C_{18}$ reversed-phase cartridge. It was thereupon found that the recovery of caffeine by the MIPbased sorbent used in this work was almost two and four times greater than that by a commercially available $\mathrm{C}_{18}$ material. A quantitative analysis was conducted by high performance liquid chromatography (HPLC) using a $\mathrm{C}_{18}$ column $(5 \mu \mathrm{m} .250 \times 4.6 \mathrm{~mm})$ with methanol/water $(40 / 60 . \% \mathrm{v} / \mathrm{v})$ as the mobile phase at a flow rate of $0.5 \mathrm{~mL} / \mathrm{min}$.
\end{abstract}

Key Words : Solid-phase extraction. Molecular imprinted polymer. Caffeine. Catechin compounds

\section{Introduction}

The technique of molecular imprinting consists of the selfassembly of a functional monomer and a template molecule in solution followed by co-polymerization of the functional monomer and an excess of an appropriate crosslinking monomer. After removal of small molecules. the resulting network polymer exhibits significantly higher affinity for the molecules used as the template than for similar molecules. including closely related isomers. ${ }^{1-3}$ MIP has been applied to chiral separation. ${ }^{4.5}$ solid extraction. ${ }^{6.7}$ biomimic sensor. ${ }^{8.9}$ and membrane separation. ${ }^{10.11}$ While MIP can be prepared by both the covalent and the non-covalent method. the latter has been widely used in recent years owing to its relatively' simple procedure." This utility, which is based on their shape. size. and functionality selectivity. strong affinity on rebinding target compounds. and significantly low cost for preparation and workability in organic solvents. calls for finding a proper template to improve their selectivity and affinity. ${ }^{13}$

In recent years. solid-phase extraction involving a molecular imprinted polymer (MISPE) has been successfully' applied to biological and environmental samples ${ }^{1+1 \S}$ owing to its features of high selectivity. ease of synthesis. low cost for preparation and workability under different conditions. especially with harsh $\mathrm{pH}$ and organic solvents. The use of MIP in SPE is advantageous mainly when selective extraction must be performed and the commonly used sorbents lack selectivity. MISPE allows not only the analyte to be pre-concentrated but also the other compounds present in the sample matrix to be removed. ${ }^{19}$

Recent studies have shown that green tea confers great beneficial effects to the health of consumers. including effects of reduction of cholesterol. depression of hypertension, antioxidation. anti-microbial, and protection against cardiovascular disease and cancer. ${ }^{20}$ The major constituents in green tea are polysaccharides, flavonoids. vitamins B. C. and E. Ramino butyric acid. catechin compounds such as ECG EGCG $+C . E G C$ and EC. and fluoride. Among these components. catechin compounds have attracted attention for their strong sulfated effect and anti-cancer function. While the pharmaceutical activities of the components have been studied. ${ }^{21-23}$ new efforts with high purity and selectivity are still under progress. During the past few years. xanthines including caffeine have been researched as a potential template for MIP. ${ }^{24.25}$ The application of MIP as a selective sorbent material has been successfully performed in solid phase extraction. but there have been relatively few reports on it application in extracting certain active components directly from natural plants. ${ }^{2 t}$

In this work, using a caffeine template protocol. MIP was prepared for the extraction of caffeine and some catechin compounds from green tea. Commercial $\mathrm{C}_{18}$ particles and MIP were used as SPE sorbents for extraction of caffeine and catechin compounds from green tea.

\section{Experimental Section}

Chemicals. The green tea used in this experiment was cultivated at Bosung (Chonnam. Korea). The standard chemicals of caffeine methacrylic acid (MAA), (-)epigallocatechin (EGC). (+) Catechin (+ C). (-)epicatechin (EC). and $(-)$ epigallocatechin gallate $(\mathrm{EGCG})$ were purchased 
from Sigma (ST Louis, MO. USA). $\alpha \alpha \alpha^{\prime}$-Azobis (isobutyronitrile) (AIBN) was obtained from Junsei Chemical Co.. Ltd. (Japan). Ethylene glycol dimethacrylate (EGDMA) was from Fluka (Buchs. Switzerland). All the above reagents were used directly without further treatment. Acetonitrile and methanol were all of HPLC grade and from Duksan Pure Chemical Co. LTD (Ansan. Korea). Acetic acid (analytical grade) was from Oriental Chemical Industries (Incheon. Korea). Doubly distilled water was filtered by a decompressing pump (Division of Millipore. Waters) and filter (FH-0.5 $\mu \mathrm{m})$.

Polymer preparations. The following were added to a $250 \mathrm{~mL}$ two-neck glass flask: $5 \mathrm{mmol}$ of the monomer (MAA) $30 \mathrm{mmol}$ of the crosslinker (EGDMA) $0.12 \mathrm{~g}$ of the initiator (AIBN). $9 \mathrm{~mL}$ of the porogen $(\mathrm{ACN})$, and 1 mmol of the template (caffeine). The reaction mixture was subjected to supersonication for $10 \mathrm{~min}$. sparged with helium for 10 min to remove oxygen. and then vacuumed for $10 \mathrm{~min}$ and sealed under vacuum. Polymerization was performed in a water bath that was held at $60^{\circ} \mathrm{C}$ for $24 \mathrm{hr}$. After the polymerization. the bulk polymer was removed from the reaction flask and put into an oven for drying. The dried polymer was grounded into particles and passed through a $35 \mu \mathrm{m}$ sieve; small particles were removed by repeated sedimentations with water. By these procedures. particles of 25-35 $\mu \mathrm{ml}$ were collected. $\mathrm{C}_{18}$ particles were purchased from Alltech (Deerfield. IL. U.S.A.).

HPLC analysis. HPLC was used for a quantitative analysis of the SPE results. The liquid chromatography system contained a Waters $600 \mathrm{~s}$ Multisolvent Delivery System and a Waters 616 pump (Waters. Milford. MA. U.S.A.), a detector of Waters 2487 Dual Absorbance (Waters. Milford. MA. U.S.A.), and a Rheodyne injection valve ( $20 \mu \mathrm{L}$ sample loop). Millennium 3.2 (Waters. Milford. MA. U.S.A.) was used for data acquisition. Quantitative determination was based on a $\mathrm{C}_{18}$ column $(5 \mu \mathrm{m}, 250 \times 4.6 \mathrm{~mm}$ from Rstech corporation), where methanol/water $(40 / 60 . \% \mathrm{v} / \mathrm{v})$ was the mobile phase at the flow rate of $0.5 \mathrm{~mL} / \mathrm{min}$. and the injection volume $20 \mu \mathrm{L}$, and the UV wavelength was set at 270 num.

Solid-phase extraction. Commercial SPE cartridges were emptied from their packing materials. The cartridge tube and frits were then thoroughly cleaned and dried. About $200 \mathrm{mg}$ of the corresponding polymers was packed dry in the cartridges and the upper first was placed on top. The $C_{18}$ SPE cartridge (with $200 \mathrm{mg}$ packing material) used in this study were purchased from Alltech (Deerfield, IL, U.S.A). Before the extraction. the MIP cartridges were treated with methanol/acetic acid $(90 / 10 . \% \mathrm{v} / \mathrm{v}) .4 \times 3 \mathrm{~mL}$. followed by methanol $4 \times 3 \mathrm{~mL}$. C $1 \&$ was treated only by methanol $4 \times 3$ $\mathrm{mL}$. The extraction process involved loading the SPE cartridges with caffeine (concentrations are $2 \mathrm{~g} / \mathrm{mL}$ ) or a solvent extraction sample of green tea. (the loading volumes were $1 \mathrm{~mL}$. respectively) washing with $1 \mathrm{~mL}$ methanol, and eluting with a $1 \mathrm{~mL}$ mixture of methanol and acetic acid $(90 /$ 10. $\% \mathrm{v} / \mathrm{v}$ ). Fractions were collected and exaporated to dryness. The residues were reconstituted to solution with
Table 1. Calibration equations of caffeine and catechin compounds

\begin{tabular}{ccc}
\hline Compound & Equation & \multicolumn{1}{c}{$\mathrm{r}^{2}$} \\
\hline Caffeine & $y=1 \times 10^{-6} \mathrm{x}-2.1039$ & 0.9763 \\
$(+) C$ & $y=7 \times 10^{-7} \mathrm{x}-1.1884$ & 0.9956 \\
EC & $y=5 \times 10^{-7} \mathrm{x}-2.6806$ & 0.9777 \\
EGCG & $y=1 \times 10^{-6} \mathrm{x}-2.4038$ & 0.9775 \\
EGC & $y=5 \times 10^{-6} \mathrm{x}-1.0883$ & 0.9834 \\
\hline
\end{tabular}

$\mathrm{x}$ : peak area $\left(\mathrm{mAU} \mathrm{ASec}^{*}\right), y$ injection volume of catechin compounds in the water $(\mu \mathrm{L})$

$100 \mu \mathrm{L}$ methanol and analyzed by HPLC. The calibration curves were constructed to measure the amount of caffeine and four catechin compounds. The linearity is shown in Table 1. $y$ is the injection volume of caffeine and five catechin compounds in water $(\mu \mathrm{L})$ and $\mathrm{x}$ represents the peak area (mAU*sec). $5 \mu \mathrm{L}, 10 \mu \mathrm{L} .15 \mu \mathrm{L}$ and $20 \mu \mathrm{L}$ volumes of caffeine and catechin compounds were injected. For each experimental condition, at least two injections were made in the reproducibility. and the average values are listed in Table 1. The regression coefficients of straight lines for the caffeine and four catechin compounds were above 0.97 .

Solvent extraction from green tea. $5 \mathrm{~g}$ of green tea was extracted for 4 hrs with $150 \mathrm{~mL}$ doubly distilled water at 50 ${ }^{\circ} \mathrm{C}$ with stirring. The extract was filtered by a $0.2 \mu \mathrm{m}, 25 \mathrm{~mm}$ syringe filter (Alltech. Deerield. IL. U.S.A.) and then diluted with water ( $1 \mathrm{~mL}$ extract from green tea $+9 \mathrm{~mL}$ distilled water) and subsequently applied for loading on the SPE cartridge.

Caffeine content. Caffeine content in green tea was prepared via several steps. First. $0.5 \mathrm{~g}$ of green tea was extracted with $150 \mathrm{~mL}$ doubly distilled water at $50^{\circ} \mathrm{C}$ with stirring for 4 hrs. Second, the refuse of green tea was extracted with $150 \mathrm{~mL}$ doubly distilled water at $15-85^{\circ} \mathrm{C}$ with stirring for $24 \mathrm{hrs}$. Third. the refuse of green tea was extracted with 150 $\mathrm{mL}$ doubly distilled water at $15-85^{\circ} \mathrm{C}$ with stirring for 48 hrs. Fourth. the refuse of green tea was extracted by $150 \mathrm{~mL}$ doubly distilled water at $15-95^{\circ} \mathrm{C}$ with stirring for 72 lurs. Fifth the refuse of green tea was extracted by $150 \mathrm{~mL}$ HPLC grade of methanol with sonification at a room temperature for $1 \mathrm{hr}$. The extracts were filtered by a $0.2 \mu \mathrm{m}, 25 \mathrm{~mm}$ syringe filter (Alltech. Deerield. IL. U.S.A.). The extracts were then injected to the analytical HPLC. The obtained chromatograms were used for quantitative determination of caffeine in green tea.

\section{Results and Discussion}

Caffeine MIP was synthesized and prepared in this work Commercial $\mathrm{C}_{18}$ particles with the above polymers were packed into SPE cartridges for investigation of the effect of MISPE. Sorbent-analyte interactions fall into three categories non-polar, polar, and ionic. Non-polar sorbents are generally selected for extracting tri-azines from water. By contrast. degradation products. which contain very polar functional groups such as hydroxyl, carbonyls. amines. and sulfhydryls. need polar sorbents. Polar sorbents exhibit a strong tendency 
(A) $\mathrm{C}_{18}$

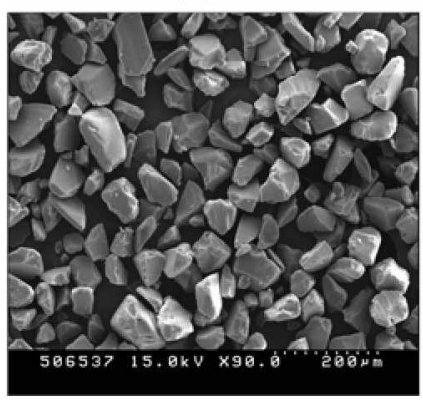

(B) MIP

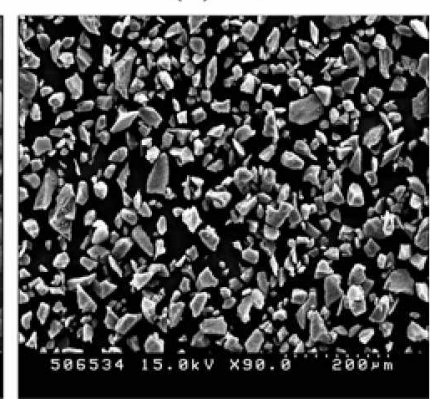

Figure 1. SEM of $\mathrm{C}_{18}$ and MIP particles. (IA) Commercial $\mathrm{C}_{18}$ particles, (B) MLP particles through the $32 \mu \mathrm{m}$ sieve.)

to form hydrogen bonds. The retention of polar compounds on polar sorbents is facilitated by non-polar solvents. Analytes that are capable of forming cations include amines: analy'tes with the potential to form anions. including carboxylic. sulfonic acids and phosphates. In order to facilitate the occurrence of ionic interactions between sorbents and analyte. an anionic sorbent should be selected to retain cations. while a cationic sorbent is needed to retain anions. For a maximum retention. $\mathrm{pH}$ of the matrix should be adjusted to $2 \mathrm{pH}$ units below the $\mathrm{p} K_{\mathrm{a}}$ of the cation and $2 \mathrm{pH}$ units above the $\mathrm{p} K_{\mathrm{a}}$ of the anion. ${ }^{27}$ ln comparison with traditional stationary phase extraction materials. a unique property of MIP is their lock-key relationship with the target molecule. and hence their selectivity can be predetermined. MISPE is a well-recognized technique for the selective extraction and pre-concentration of analytes present at low levels in chemically complex samples. ${ }^{25}$ The process consists of loading. washing. and elution of caffeine processing with caffeine MIP. In the elution process. a larger amount of caffeine is eluted than in the loading and washing process. It has been shown that the MISPE can selectively recognize the template molecule among other structurally related molecules. The commercial $C_{1}$ particles, MIP was used for extraction of caffeine standard $(0.2 \mathrm{mg} / \mathrm{mL})$ by SPE. respectively' SEM micrographs of $C_{18}$ particles and MIP are shown in Figures l(A) and (B), respectively. Although the effect of particle size on the performance of separation is important. it was not considered here and will be left for future work. Generally. MIP exhibits a better molecular recognition in the solvent used as porogen during polymerization. It was revealed that the selective binding of the template to the MIP is enhanced under conditions similar to those observed during the molecular self-assembly in the polymerization mixture.

Caffeine and some catechin compounds were extracted from green tea by MISPE. The SPE extraction of green tea followed the same load-wash-elute procedure as employed in the SPE extraction of caffeine. However. an aqueous sample of green tea was used in the loading step. Methanol and a mixture of methanol/acetic acid $(90 / 10 . \% \mathrm{v} / \mathrm{v})$ were used as the washing and eluting solvents. respectively. Figure 2 shows the chromatogram of an extract of caffeine and four catechin compounds from green tea with $C_{18}$ and MIP sorbents in the washing step. Coniparing Figure 2(A)
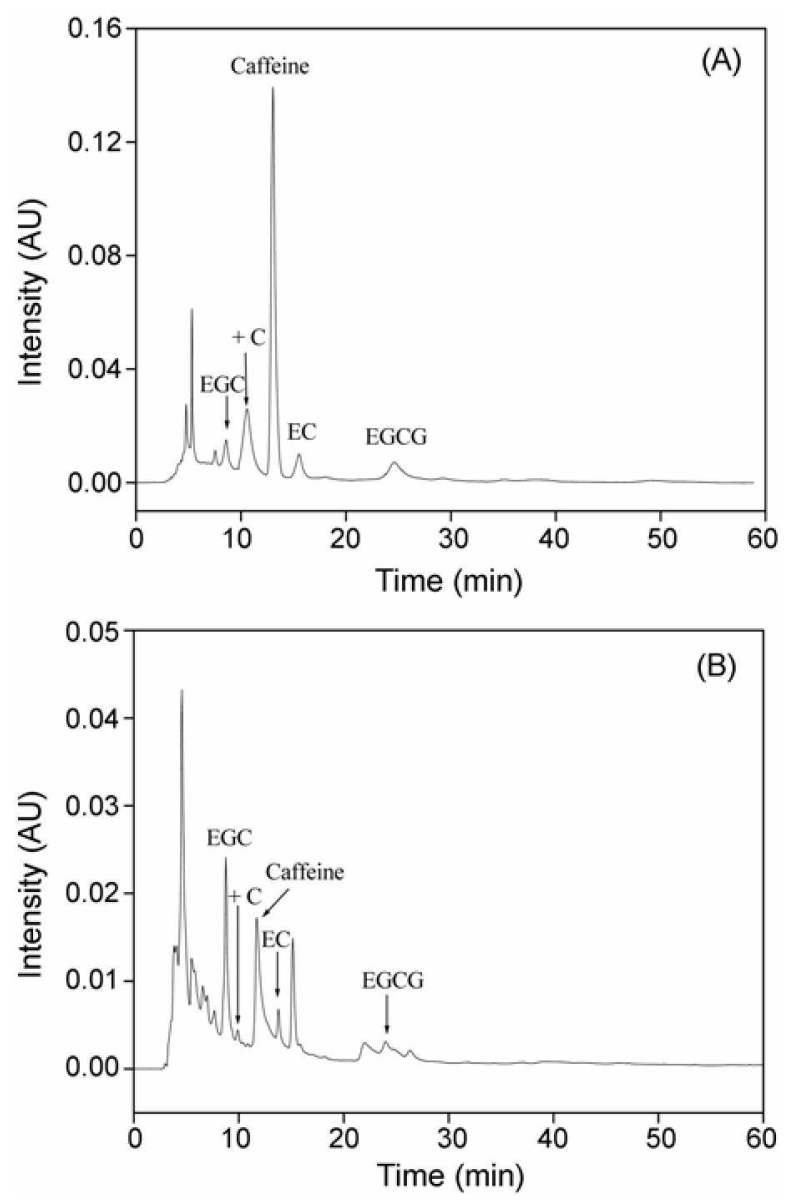

Figure 2. Chromatogram of solid-phase extraction of cafteine from green tea. ((A) Washing in $C_{18}$, (B) Washing in MIP)

and $(B)$. it is found that the caffeine and catechin compounds were extracted with the MIP sorbent. Most compounds were eluted through the washing process. including caffeine and catechin compounds. with $\mathrm{C}_{\mathrm{f}}$ s sorbents. Comparing the aforementioned sorbents. with the MIP sorbents. very small of amounts of the caffeine were eluted for loading step (Table 2). Table 2 shows the results of solid-phase extraction of caffeine and catechin compounds from green tea with different sorbents. In the loading step the amount of caffeine eluted from the $\mathrm{C}_{18}$ cartridge was $60.86 \mu \mathrm{g} / \mathrm{g}$. while 40.05 $\mu \mathrm{g} / \mathrm{g}$ were obtained for the MIP cartridge. respectively. For each experimental condition, at least two injections were made in order to confirm the reproducibility. and the average values are listed in Table $2.14 .58 \mu \mathrm{g} / \mathrm{g}$ of $+\mathrm{C}$ of were extracted for the C.18 particles. In the washing step. the amount of caffeine from the $C_{18}$ cartridge was $5296.06 \mu \mathrm{g} / \mathrm{g}$. while $1368.30 \mu \mathrm{g} / \mathrm{g}$ was obtained from the MIP cartridge. respectively. In the elution step. $7979.12 \mu \mathrm{g} / \mathrm{g}$ of caffeine was eluted from MIP particles.

The caffeine content in green tea was calculated. The extracted amounts of caffeine from green tea were 20.61 . 0.37 .0 .028 .0 .00 and $0.00 \mathrm{mg} / \mathrm{g}$. from the first step to fifth step. respectively. Hence the caffeine content in green tea was $21.01 \mathrm{mg} / \mathrm{g}$. The recovery of caffeine from green tea was 25.50 and $44.69 \%$ through the $C_{18}$ and MIP cartridge, 
Table 2. Solid-phase extraction of caileme and catechun compounds from green tea with different sorbents

\begin{tabular}{|c|c|c|c|c|c|c|c|c|c|c|}
\hline Sorbents & & & $\mathrm{C}_{18}$ & & & & & feine MI & & \\
\hline Compontents & $+\mathrm{C}$ & Cafteine & $\mathrm{EC}$ & $E G C$ & $\mathrm{EGCG}$ & $+\mathrm{C}$ & Caffeine & $\mathrm{EC}$ & $\mathrm{EGC}$ & $\mathrm{EGCG}$ \\
\hline Loading ( $\mathrm{ug} / \mathrm{g}$ ) & 14.58 & 60.86 & 0.00 & 0.00 & 0.00 & 0.00 & 40.05 & 0.00 & 0.00 & 0.00 \\
\hline Washing (ug/g) & 2241.00 & 5296.06 & 756.71 & 253.55 & 183.38 & 8.70 & 1368.30 & 640.23 & 862.31 & 28.14 \\
\hline Elution ( $\mathrm{ug} / \mathrm{g}$ ) & 0.00 & 0.00 & 0.00 & 0.00 & 0.00 & 0.00 & 7979.12 & 0.00 & 0.00 & 0.00 \\
\hline Total (ug/g) & 2255.58 & 5356.92 & 756.71 & 253.55 & 183.38 & 8.70 & 9387.47 & 640.23 & 862.31 & 28.14 \\
\hline
\end{tabular}

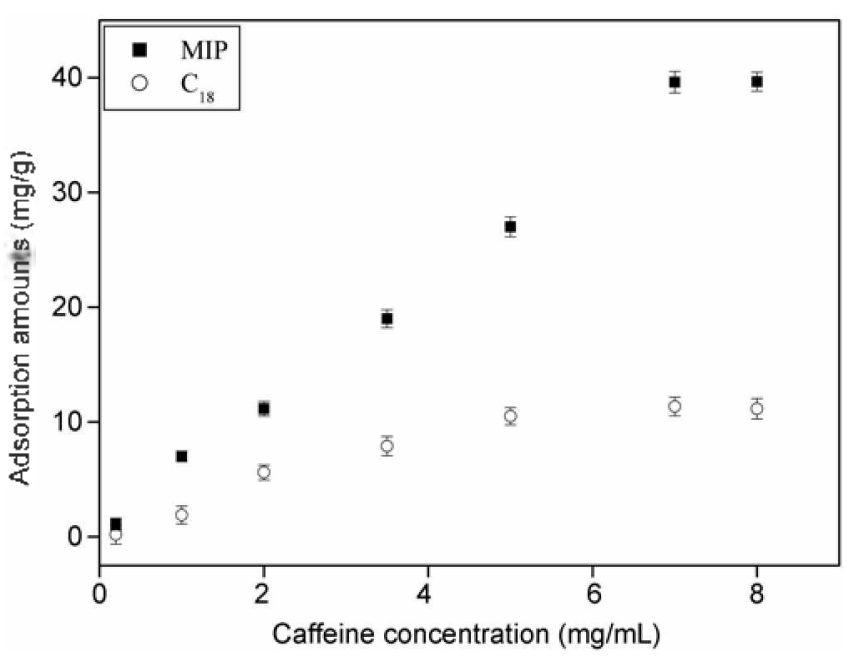

Figure 3. Comparison of the adsorption amounts of caffeine by MIP and $\mathrm{C}_{18}$ particles.

respectively, and the values are listed in Table 2 . In the elution processing. caffeine was not detected on the $\mathrm{C}_{1 \delta}$ cartridge. On the other hand, a considerable amount of caffeine was eluted in elution process from the MIP cartridge. The main component adsorbed on MIP was found to be caffeine with content and purity of $7979.12 \mu \mathrm{g} / \mathrm{g}$ and $84.83 \%$, respectively: It is known that the selective binding sites are mainly formed by lydrogen bonding or electrostatic force during self-assembly between the template and monomer. while the other part of the MIP surface remains non-selective. i.e. hydrophobic. In this case. when the aqueous sample was applied during the loading step. the selective binding of the MIP was suppressed and the MIP behaved as a reversed-phase material. As water is a high polar solvent and shows strong hydrogen bonding ability. similar results were observed when SPE materials were employed. On the other hand. one can conclude that the MISPE materials used in this work are comparable with the conventional $\mathrm{C}_{18}$ particles. Methanol or acetonitrile containing a small percentage of acid, such as trifluoroacetic acid ${ }^{29}$ and acetic acid ${ }^{30}$ have been used as eluting solvents to recover the tenplate from the MISPE cartridge. All of these studies demonstrate that the high polar solvent can interfere with the specific binding. i.e.. hydrogen bonding or electrostatic interactions between the template and the polymer. Otherwise. the hydrophobic interactions can be enhanced. This may account for the low recovery on the $\mathrm{C}_{18} \mathrm{SPE}$ column in this work.

The extracted amounts of caffeine by' molecular imprinted polymer and $\mathrm{C}_{1 \leqslant}$ particles at the solid-phase cartridges were measured by varying concentrations. after the equilibrium adsorptions of caffeine on MIP and $C_{1 \%}$ particles were attained. Comparing the amount of caffeine by MIP sorbent with that by $\mathrm{C}_{1 \times}$ particles. similar trends with a larger concentration of caffeine up to $5 \mathrm{mg} / \mathrm{mL}$ were observed. and more caffeine was adsorped on the stationary phases. Above the caffeine concentration. on the three stationary phases. the adsorbed amounts were asymptotic to the saturated values. Figure 3 shows the experimental results of the adsorption amounts of caffeine on the molecular imprinted polymer and $\mathrm{C}_{\mid:}$sorbents. The results indicate that the imprinted molecularly polymer shows a higher affinity to the target molecule of caffeine than the $C_{1 \times}$ particles. That is. the caffeineimprinted polymer possesses a higher saturation capacity for the template than that of molecule on the $\mathrm{C}_{\mid \leqslant}$particles.

\section{Conclusions}

In this work, a caffeine molecular imprinted polymer was prepared. The caffeine MIP showed higher selectivity to the caffeine molecules. MIP and $C_{1}$ sorbents were used for solid-phase extraction of caffeine and four catechin compounds from green tea. When applied to the solid-phase extraction of green tea. the MIP materials behaved as packing materials reversed-phase HPLC. and the extraction results were comparable with those of conventional $C_{1}$ particles. The caffeine content in green tea was $21.01 \mathrm{mg} / \mathrm{g}$. In the elution processing nearly pure caffeine was detected on the caffeine MIP cartridge. It was observed that higher affinity and recovery of caffeine could be obtained on the MIP cartridge than on the commercial $\mathrm{C}_{18}$ materials. Synthesized and prepared caffeine MIP could selectively extract and remove the caffeine and some catechin compounds from green tea. The molecularly imprinted polymer showed a higher affinity to the target molecule of caffeine than did the $C_{18}$ particles. Furthermore. MIP can be used for the extraction of specific components from crude plants by SPE.

Acknowledgement. This work was funancially supported by the Center for Advanced Bioseparation Technology and High-Purity Separation Lab. Department of Chemical Engineering. Inha Univerșity. Korea.

\section{References}

1. Chen. W: Liu, F.; Zhang, X; Li, K. A.: Tong, S. Talanta 2001, 55. 29. 
2. Zhang. T.: Liu. F.: Chen. W: Wang. J.: Li. K. Anallyica Chinica Acta 2001.450 .53 .

3. Zheng. N.: Li. Y. Z.: Chang. W. B.: Wang. Z. M.: Li. T. J. Anatotica Chimica Acta 2002. 152.277.

4. Ramstrom, O.: Yu, C.: Mosbach, K. J. Mol. Recogniti. 1996. $9(5 ; 6) .691$.

5. Schweitz. L.: Andersson. L. I; Niilsson. S. Afal. Chem. Acta 1997. 69(6). 1179.

6. Blomgre. A.: Berggren. C.: Holmberg. A.: Larsson. F.: Sellergre. B.: Ensing. K. J.Chromatogr .A 2002. 975.157

7. Wang. D. X.: Hong. S. P. Row, K. H. Konam J. Chem, Eng. 2004. $21(4) .853$.

8. Yano, K.: Karube. I. Trends in Anal. Chem. 1999, 18(3). 199.

9. Snowden. T. S.: Anslyn. E. V. Current Opinton in Chem. Bio. 1999. 3(6). 740 .

10. Ulbricht. M. J. Chromatogr: B 2004. 804. 113

11. Park. J. K.: Kim. S. J. Korean J. Chem. Eng. 2004. 21 (5). 994.

12. Wang. D. X: Hong. S. P.: Row. K. H. Buh. Koream Chem. Soc. $2004,25(3) .357$

13. Jin. Y: Row. K. H. Koren J. Chem. Eng. 2005. 22(2). 264.

14. Caro. E.: Marce. R. M.: Cormack. P. A. G.: Sherrington. D. C.: Borrull. F. J. Chromtatogr A 2003.995. 233.

15. Stanley, G. W.: Edward. P. C. L.; Paul, M. M. J. Phamacentical and Biomedical Analysis 2004. 36, 483.

16. Sherry. Y. F.: Edward. P. C. L.: Ewa. D. Z: Susan, S. J. Chronatogr \& 2004. 1027. 155.

17. Zhu. X: Yang. J.: Su. Q.: Cai. J.: Gao. Y. J. Chrontatogr. A 2005
1092. 161

18. Jung. M. W.: Kim. K. P.: Cho. B. Y: Paeng. I. R.: Lee. D. W: Park. Y. H.: Paeng. K. I. Bull. Korean Chent. Soc. 2006. 27(1). 77.

19. Caro. E.: Marce. R. M.: Borrull, F.: Cormack, P. A. G.: Sherrington, D. C. Trends in Analytical Chenistry 2006. 25(2), 143.

20. Zuo. Y.: Chen. H: Deng. Y. Talanta 2002. 57. 307.

21. Melissa. K.: George. L. Mntation Resecurch 2000. 459.211.

22. Kang. J. H.: Chung. S. T.: Row. K. H. J. Ind. Eng. Chent 2002. $8(4) .354$

23. Cheong. W. J.; Park. M. H.: Kang, G. W.: Ko, J. H.; Seo, Y. J. Bull. Korean Chem. Soc. 2005. 26(5),747.

24. Mullett. W. M. Lai. E. P. C. I. Pham Bioned Anal. 1999. 21. 835.

25. Kobayashi. T: Murawaki. Y.: Reddy. P. S.: Abe. M.: Fujii. N. Anafitica Chintica Acta 2001. +35. 141 .

26. Xie. J; Zhu, L; Luo. H; Zhou. L.: Li, C $;$ Xu, X. J. Chronatogr: A 2001,934. 1 .

27. Hassan. S.; Roger, J.: Bernard. R. $J$. Chromatogr: A 2000. 885. 217.

28. Zurutuza. A.: Bayoudh. S.: Cormack. P. A. G: Dambies. L.: Deere. J.: Bischoff. R.: Sherrington. D. C. Analytica Chimica Acta 2005. 542,14 .

29. Olsen. J.: Martin. P: Wilson. I. D.: Jones. G. R. Analyst 1999. $12+(4) .467$

30. Baggiani. C.: Trotta. F.: Giraudi. G.: Giovannoli. C.: Vanni. A. Anal. Comm 1999. 36(7). 263. 